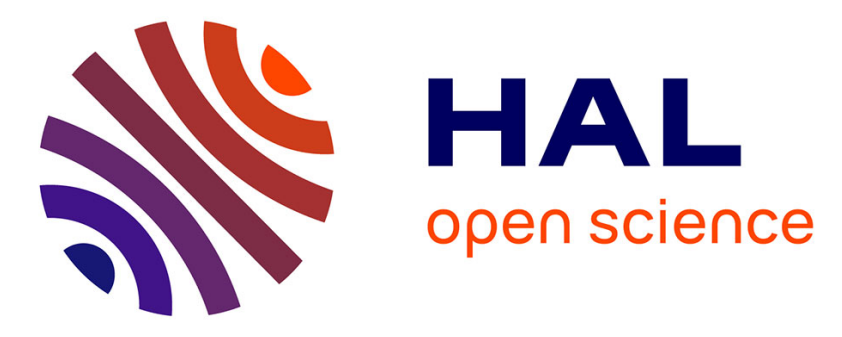

\title{
Magnetic Resonance Imaging guided cardiac radiofrequency ablation
}

Valery Ozenne, Solenne Toupin, Pierre Bour, Aurélie Emilien, F. Vaillant, Baudouin Denis de Senneville, Pierre Jaïs, Jenny Benois-Pineau, Pascal Desbarats, Bruno Quesson

\section{To cite this version:}

Valery Ozenne, Solenne Toupin, Pierre Bour, Aurélie Emilien, F. Vaillant, et al.. Magnetic Resonance Imaging guided cardiac radiofrequency ablation. Innovation and Research in BioMedical engineering, 2015. hal-01116816

\author{
HAL Id: hal-01116816 \\ https://hal.science/hal-01116816
}

Submitted on 15 Feb 2015

HAL is a multi-disciplinary open access archive for the deposit and dissemination of scientific research documents, whether they are published or not. The documents may come from teaching and research institutions in France or abroad, or from public or private research centers.
L'archive ouverte pluridisciplinaire HAL, est destinée au dépôt et à la diffusion de documents scientifiques de niveau recherche, publiés ou non, émanant des établissements d'enseignement et de recherche français ou étrangers, des laboratoires publics ou privés. 
Magnetic Resonance Imaging guided cardiac radiofrequency ablation

Valéry Ozenne ${ }^{1}$, Solenn Toupin ${ }^{1}$, Pierre Bour ${ }^{1}$, Aurélie Emilien ${ }^{1}$, Fanny Vaillant ${ }^{1}$, Baudoin Denis de Senneville ${ }^{2}$, Pierre Jaïs ${ }^{1}$, Jenny Benois-Pineau ${ }^{3}$, Pascal Desbararts ${ }^{3}$, Bruno Quesson ${ }^{1}$

1 L'Institut de Rythmologie et Modélisation Cardiaque, Pessac, France,

2 Mathematical Institute of Bordeaux, Bordeaux, France,

3 LaBRI, CNRS/Université Bordeaux, Talence, France 
keywords

KEYWORDS: cardiac arrhythmia, RF ablation, thermal therapy, MR thermometry, image registration, motion analysis. 


\section{ABSTRACT:}

Although radiofrequency ablation has become a worldwide accepted curative therapy for the treatment of cardiac arrhythmias, there is still a lack of lesion monitoring during ablation procedure. Magnetic resonance thermometry based on the water proton frequency could give relevant information on local tissue temperature increase but requires dealing with motion and susceptibility artifacts due to the cardiac and respiratory motion. A real-time framework that overcomes these difficulties is described in this paper. The proposed approaches address both inter-scan and inplane motion using a rapid MR-acquisition sequence coupled with a robust optical-flow registration of all incoming images. The strategy was found to be robust and suitable to follow the temperature evolution in 10 healthy volunteers under free breathing conditions with a temperature standard deviation of $2.2^{\circ} \mathrm{C}$ in the cardiac muscle at an update rate of approximately $1 \mathrm{~Hz}$. Since local temperature rises due to RF ablation can lead to $\mathrm{T} 1$ and $\mathrm{T} 2$ relaxation time local variations, and thus to errors in the image-based registration process, we also analyze the performance of the algorithm during radiofrequency ablation applied under well controlled experimental conditions (no respiratory motion and cardiac triggered) using an ex-vivo perfused pig heart. Contact electrophysiological signals were recorded simultaneously with magnetic resonance imaging by combining hardware and software filtering. A working heart setup, specifically designed for this project, provided a relevant model to assess the quality of cardiac MR-thermometry. 


\section{INTRODUCTION}

Cardiovascular disease is the first cause of mortality in the whole world. In 2008, the World Health Organization reported that it is responsible for $29 \%$ of deaths, followed by infectious diseases (16.2\%) and cancers (12.6\%) (1). In the Western countries, cardiovascular disease is as deadly as cancer ( $28 \%$ vs. $30 \%$ respectively) (1). Amongst cardiovascular disease, cardiac electrical diseases are a major burden for the patients (severe morbidity and poor quality of life) and the health care system. Mechanistically simple arrhythmias (involving a unique focus or circuit) can now be cured by localized energy application (catheter ablation) with a high success rate. However, in patients presenting with persistent atrial fibrillation (AF), the extent and locations of the arrhythmogenic substrate are highly variable. The success rate of ablation procedures is in this population lower than in paroxysmal AF patients. It requires the identification of the substrate on a patient by patient basis, based on a refined analysis of detailed electrophysiological (EP) contact mapping data. In case of ventricular tachycardia (VT), radiofrequency ablation (RFA) has been proposed as an additional strategy in order to cauterize critical sites and interrupt VT circuits $(2,3)$. Several authors have recently suggested a preventive ablation for every patient before implantable cardioverter defibrillator (ICD) implantation (4). These ablation procedures are now feasible in sinus rhythm by targeting the slow conduction area. Despite significant efforts done worldwide for the fusion of different anatomical and functional information of a heart showing dysfunction, there is a clear lack of non invasive imaging during the treatment.

Magnetic resonance imaging (MRI) is nowadays extending from diagnosis to the field of real time monitoring of therapeutic procedures (e.g. MR temperature imaging for the guidance of non invasive thermo ablation with High Intensity Focused Ultrasound) (5). The importance of MRI in understanding and managing cardiac arrhythmias is still preliminary but is creating much hope for diagnosis, treatment monitoring (online computer assisted feedback to the physician) and therapy assessment (definition of a quantitative criteria for clinical endpoint).

Recent publications have demonstrated that T1-weighted images acquired immediately after the RFA allow visualization of lesion extension (6). However, there is still a clear lack of lesion monitoring during the ablation procedure, although RF ablation has become a worldwide accepted curative therapy for the treatment of cardiac arrhythmias. Initial method for monitoring RF ablations were based on the observation of tissue impedance (between the tip electrode and the return electrode positioned on the skin of the patient) changes and/or temperature measurement at the tip of the catheter during the procedure. However, these data poorly correlate with tissue temperature at distance from the contact point of the RF electrode and hardly reflect the lesion size and thus cannot be used as a predictor for complete trans-mural ablation.

Real-time lesion visualization through online thermometry requires the development of dedicated, rapid, temperature sensitive, acquisition sequences in order to be able to provide sub-second information at each cardiac cycle. From the MR-raw data, images must be reconstructed in real-time and streamed to a separate workstation (Figure 1.a). Magnitude and phase images have to be processed online to correct for motion and susceptibility related artifacts on the phase information in order to provide actual 
temperature measurement in the myocardium with minimal uncertainty $\left(<2^{\circ} \mathrm{C}\right)$. Dedicated catheter technology is required to avoid image artifacts and to provide electrophysiological (EP) recordings simultaneously to MR-thermometry and RFA. Such objective is challenging regarding the voltages emitted by the radiofrequency pulses of the MR scanner (400 V) and the RFA generator (typically 50V or higher) relative to the millivolt amplitude of the EP signals (typically 2 to $5 \mathrm{mV}$ maximal amplitude).

The objective of this paper is to summarize the recent developments of our team in this field.

\section{MATERIAL AND METHODS}

\section{Real-time MR thermometry}

Dynamic MR thermometry was performed at 1.5T MR (Avanto, Siemens Medical Solution, Erlangen, Germany) using an in house developed single-shot gradient echo planar imaging (EPI) sequence with the following parameters: 2 to 5 slices in coronal orientation (to observe main respiratory motion in the imaging plane, figure 1.b) acquired under two hundred and fifty successive heart beats (ECG triggered in diastolic phase), TE $=21 \mathrm{~ms}$, $\mathrm{TR}=180 \mathrm{~ms}$, Flip Angle $=40^{\circ}$, Field Of View $=280 \times 280 \mathrm{~mm}^{2}$, Resolution $=2.5 \times 2.5 \times 5 \mathrm{~mm}^{3}$, Acceleration Factor=2, 32 channels cardiac coil. Two saturation slabs were positioned parallel to the imaging stack for attenuating the blood signal into the cavities and two additional slabs were positioned in the phase encoding direction for field of view reduction. This imaging sequence was evaluated on 10 healthy volunteers during free breathing.

\section{Image processing pipeline}

To evaluate temperature evolution on each pixel of the magnitude image, in-plane motion due to respiration and cardiac contraction has to be compensated to the same position of reference. For this purpose, one frame of the MR acquisition was chosen as the reference image and the Black Anandan optical flow algorithm (7) was used to estimate 2D motion displacement between the reference image and each frame of the time series. The registration process had to correct both magnitude and phase image by carrying out the following steps (see Figure 1.a):

- For each acquisition the optical flow algorithm computed the displacement field $(U, V)$ between the current magnitude image and the reference magnitude image. A dedicated algorithm has been developed which integrates a physically relevant model for MR-signal and noise distributions to provide different weights for the pixels in the registration process depending on their respective SNR. For detailed description of this method, please refer to (8).

- To correct motion-related phase variations, the magnitude-based $(U, V)$ displacement field was used to register phase images in the complex domain. 


\section{Correction of susceptibility artefacts}

Respiratory motion induces magnetic field susceptibility variation due to organ motion and variation of lungs volumes, which results in local additional shift on phase images. If not corrected, susceptibility artefacts may lead to significant errors in the temperature computation (several degrees amplitude). In this work, two approaches have been implemented and evaluated on the heart of healthy volunteer: a multi-baseline method and a principal component analysis (PCA)-based phase modelling method. Each correction strategy is divided into two parts: a learning step followed by the interventional procedure.

\section{Multi-baseline method}

During the learning step, the multi-baseline method $(9,10)$ collects phase information at various stages of the respiratory cycle: the magnitude images and their respective coregistered phase images are acquired and stored in a library. During intervention, the current temperature map was computed using the proton resonance frequency (PRF) relation:

$$
\Delta T=\frac{\varphi-\varphi_{R E F}}{\gamma \cdot \alpha \cdot T E \cdot B o}
$$

Where $\Delta T$ is the temperature variation, $\varphi$ and $\varphi_{R E F}$ respectively the current and reference phase images, $\frac{\gamma}{2 \pi}=42.59 \mathrm{MHz} / \mathrm{T}$ is the proton gyromagnetic ratio, $\alpha=$ $-0.0094 \mathrm{ppm} /{ }^{\circ} \mathrm{C}$ is the PRF temperature coefficient, $T E$ is the echo time, $B_{0}=1.5 \mathrm{~T}$.

The baseline subtraction $\left(\varphi-\varphi_{R E F}\right)$ is then performed by matching the image acquired during intervention with the closest corresponding data stored in the library in order to mitigate susceptibility related phase.

\section{PCA-based phase modelling method}

This approach collects optical flow fields from registration during the learning step and builds a parameterized flow model using a PCA algorithm:

During the learning step, phase images and magnitude-based displacement fields $(U, V)$ were stored and used to evaluate the relation between respiratory motion and resulting phase variation. As a first step, a PCA algorithm was used to compute an orthonormal basis $B_{i}$ that depicts the main components of the motion. For each acquisition image, a set of motion descriptors $D i$ was calculated to represent its position in the respiratory cycle. The overall magnetic field variations were approximated as the sum of linear phase changes of each motion descriptor on a pixel-by-pixel basis giving a parameterized magnetic field model (11).

During the interventional procedure, the $N$ largest PCA-based motion descriptor were estimated from the current flow field and used to reconstruct magnetic field distribution from the parameterized model.

$$
\varphi_{\mathrm{REF}}(\mathrm{x}, \mathrm{y})=\sum_{\mathrm{i}=0}^{\mathrm{N}-1} \mathrm{D}_{\mathrm{i}} \cdot \mathrm{B}_{\mathrm{i}}(\mathrm{x}, \mathrm{y})+\mathrm{B}_{\mathrm{N}}
$$


Therefore, temperature map is calculated from PRF relation using this synthetic phase of reference $\varphi_{R E F}$.

\section{Statistical analysis}

To assess the quality of the temperature measurements, the temporal standard deviation of temperature (TSD or $\sigma \mathrm{T}$ ), reflecting precision and stability, was calculated for each pixel during the interventional procedure (two hundred dynamics) without temporal filtering nor data removal. The distribution of TSD values was analysed on a manually drawn $\mathrm{ROI}$ surrounding the ventricle by measuring the temperature levels corresponding to three percentages of the distribution: Q1 (25\% of the distribution of TSD values), Q2 (median) and Q3 (75\% of the distribution of TSD values). The work-flow was implemented on an external workstation using 2 Xeon processors $(2.5 \mathrm{GHz}, 4$ cores, INTEL Santa Clara, CA, USA) with 32 $\mathrm{GB}$ random-access memory. Implementation was written in $\mathrm{C}++$ and makes use of the Armadillo linear algebra library (12).

\section{Ex vivo working heart setup}

In order to estimate the precision of MR thermometry in absence of respiratory motion, an ex vivo working heart model of pig was developed. Each animal (Large White $x$ Landrace, $\sim 40 \mathrm{~kg}, \mathrm{~N}=3$ ) was premedicated with Ketamine $(20 \mathrm{mg} / \mathrm{kg})$ and Calmivet $(1 \mathrm{mg} / \mathrm{kg})$, and then anesthetized with pentobarbital $(10 \mathrm{mg} / \mathrm{kg})$. After induction of anesthesia, animals were intubated and ventilated, and received an injection of heparin $(2.5 \mathrm{mg} / \mathrm{kg})$. Anesthesia was maintained with ketamine and midazolam $(40 \mathrm{mg} / \mathrm{kg} / \mathrm{h}$ and $2 \mathrm{mg} / \mathrm{kg} / \mathrm{h}$ respectively). During anesthesia, the thorax was opened and the hearts were extracted rapidely (13). Then, the aorta, pulmonary veins and artery were cannulated to perfuse the heart ex vivo.

After cannulas were positioned, hearts were reperfused in the Langendorff mode at $60 \mathrm{mmHg}$ of pressure perfusion for 15-20 minutes to wash out cardioplegic solution, rewarm gradually hearts and finally recover a stable ex vivo cardiac function. Standard introducers (11 Fr, St Jude Medical) were adapted and included into the tubing connected to the different heart chambers for inserting MR-compatible catheters for Pacing, EP recordings and RFA. Pressure sensors were inserted into the left ventricle (LV) to monitor the heart function. Full details of the setup and assessment of its MR compatibility can be found in Ref. (13).

The left ventricular ejection fraction (LVEF) was assessed by MRI measurements and compared to in vivo data obtained on the same animal before heart removal using identical methodology. LVEF was assessed on retrospectively gated CINE short axis images acquired using a multi slice balanced-SSFP sequence with $T E=1.43 \mathrm{~ms}, \mathrm{TR}=3.7 \mathrm{~ms}$, Slices $=12$, Cardiac Phases $=20$, Flip Angle $=66^{\circ}$, Resolution $=0.9 \times 0.9 \times 2 \mathrm{~mm}^{3}$. Data analyses were performed using ARGUS software (Siemens Medical Solution, Erlangen, Germany).

RF-ablation device and MR compatible EP 
A MR-compatible RFA catheter (Biosense Webster, Diamond Bar, U.S.A.) was inserted into the left ventricle and the tip electrode was connected to a RF generator (Stockert Medical Solution, Freiburg, Germany) located outside the Faraday cage.

One additional MR-compatible catheter was inserted into the right atria (RA). Cables from each catheter (except the tip electrode) were extended and connected to a clinical EP acquisition system Bard $^{\circledR}$ (LabSystem ${ }^{\mathrm{TM}}$ ) located outside the faraday cage. Band Reject Filters (attenuation $>40 \mathrm{~dB}$ ) tuned to the MR proton resonant frequency $(64 \mathrm{MHz}$ ) were designed and inserted into each transmission line for each electrode (typically 4 per catheter, distributed near the tip of the catheter). Rapid gradient switching during MRI acquisition induced voltages on electrocardiogram, but in the range of frequencies much higher $(\mathrm{kHz})$ than the current frequency range of the EP signals $(1-3 \mathrm{~Hz})$. The recordings were digitally filtered in post-processing with a low-pass $9^{\text {th }}$ order Butterworth filter $(170 \mathrm{~Hz}$ cutoff frequency) implemented in Matlab (the MathWorks Inc., USA).

\section{RESULTS}

\section{Ex Vivo working heart}

No technical failure was observed. The LVEF was evaluated at the reperfusion in the working mode. Ex vivo LVEF was reduced when compared to in vivo measurements (23.8 \pm $0.4 \%$ vs. $56.4 \pm 7.1 \%$ ). The hearts displayed a stable hemodynamic during 80 minutes before a slow decrease of the mechanical function over the following hour. Pacing of the RA and both ventricles was successfully tested without inducing artifacts on the MR images (Figure 2).

\section{EP during MRI}

Electrocardiograms recorded by the RA and LV catheters in absence of MRI sequence are shown on Figure $3 a$ under basal condition. During a MR thermometry sequence, exploitable signals can be recorded although artifacted signals can be observed during the systole (ECG gated sequence) with amplitudes larger than the maximal physiological voltage (Figure $3 \mathrm{~b}$, top traces). The unfiltered and filtered signals from two different dipoles (distalto-second and distal-to-fourth electrodes) from the RA catheter are presented. The amplitude of the artifacted voltage varies as a function of the selected dipoles and can be attenuated by the low-pass digital filter, restoring exploitable EP recording simultaneous to MR-thermometry.

\section{MR thermometry during RFA}

To evaluate temperature accuracy during heating, a RFA was performed on an isolated working porcine heart. A compatible RF catheter was positioned into the left ventricle in contact with a papillary muscle (Figure 1.e right). Ablation was run at $20 \mathrm{~W}$ during $60 \mathrm{~s}$. The evolution of temperature near catheter tip is reported in Figure 1.e (left) using PCA-based MR thermometry without any filtering method. A hyperthermia of $12^{\circ} \mathrm{C}$ was observed with a TSD before heating of $1.30^{\circ} \mathrm{C}$. 


\section{Results on In Vivo Data sets}

Temperature stability after compensation of motion-related susceptibility changes with the two methods, multi-baseline and PCA-based phase modelling was analysed on the heart of ten healthy volunteers during free breathing. For both methods, forty images were acquired during the learning step and used to compute correction on the two hundred following images of the time series, which corresponds to an acquisition time of three minutes. MR acquisition was triggered on ECG, so that two slices per heart beats were acquired during diastole. Figure 1.c compares the precision of the two methods.

On average over all volunteers, the TSD is improved with a median value (and an interquartile range, $I R Q=Q 3-Q 1)$ going from $2.7^{\circ} \mathrm{C}\left(2.5^{\circ} \mathrm{C}\right.$ respectively) with the multi-baseline method to $2.2^{\circ} \mathrm{C}\left(2^{\circ} \mathrm{C}\right)$ with the PCA-based method. These results were compared to the theoretical temperature uncertainty (dotted line on Figure 1.c) computed from the measured Signal to Noise Ratio (SNR) of magnitude images $(\sigma(\Delta T) \sim 1 / S N R)$. Over the ten human volunteers, the average SNR was evaluated to 16.7 (minimum $=9.3$, maximum $=$ 28.0) in the heart. Figure 1.d shows the TSD values of the two methods on the myocardium of the volunteer \#5, a residual uncorrected phase jump remains visible on the upper part of the myocardium. For this volunteer, $68.5 \%$ of the pixels in $\mathrm{ROI}$ remained below $3^{\circ} \mathrm{C}$ with PCA-based modelling, instead of $55.8 \%$ with multi-baseline, corresponding to an improvement of $23 \%$. This result is also observed over all in vivo data-sets with an average improvement of $21 \%$.

\section{DISCUSSION AND CONCLUSION}

In this study, we developed a dedicated MR thermometry protocol, with the objective of allowing precise temperature estimate (standard deviation of temperature below $2^{\circ} \mathrm{C}$ ) with sufficient temporal resolution ( $\sim \mathrm{Hz}$, depending on the cardiac frequency) to monitor RFA of the myocardium using dedicated MR-compatible catheters and home-made filters.

A fast acquisition sequence was designed and reconstructed images were streamed online to a dedicated image processing pipeline that allows motion correction and compensation of related susceptibility artifacts. The proposed approach addresses both inter-scan and in-plane motion without any use of echo-navigator to compensate for respiratory motion.

Two strategies of susceptibility artefacts compensation were investigated. The multibaseline approach can intrinsically only correct artefacts associated with the same positions as the ones observed in the learning phase. Although simple, this method is intrinsically less robust to spontaneous or higher amplitude motion that is expected to occur during arrhythmia. To overcome this major limitation, the PCA-based strategy was evaluated. This method of correction is not constrained to previously learned positions and can be interpolated to intermediate positions and extrapolated to larger amplitude of motion, in the limit of validity of the model. It was found to be more efficient in all examined cases and suitable to follow the temperature evolution in vivo and during ex vivo RFA. It offers a robust 
approach for motion estimation in regions prone to local signal variations on magnitude images resulting from tissue heating (signal amplitude on magnitude image may drop due to T1 increase and intra voxel spin dephasing). The possibility to extend the presented methodology to in vivo on a large animal model ablation is currently under evaluation. Temporal resolution of two slices per cardiac cycle during the ablation process appears sufficient to characterize the temporal temperature evolution. In the current implementation, we observed a latency of $1 \mathrm{sec}$ due to the processing time using two slices which allow an "almost" real-time monitoring of heating. Current work explores the possibility to incorporate GPU-based implementation for on-line processing to drastically shorten the computation time for the motion estimation algorithm (which is the most time consuming in the pipeline) and to allow larger number of slices (typically five) to be simultaneously processed in a sub-second time duration.

RFA was successfully performed without altering MR image quality using a clinical generator connected to dedicated filters. Contact EP signals could be recorded simultaneously to MRI by combining hardware and software filtering. The working heart setup specifically designed for this project provides a relevant model to assess the quality of cardiac MR-thermometry under well controlled conditions. Current work aims to improve the pacing strategy to allow induction of arrhythmia in order to test the performance of the MR-thermometry sequence under unstable physiological conditions.

MR acquisition sequences will also be improved to incorporate respiratory compensation during acquisition. Slice tracking using navigator positioned onto the diaphragm will be implemented to allow arbitrary positioning of the MR slice orientation. Such functionality may help in improving image quality (e.g. better blood signal suppression by saturation slab perpendicular to the main direction of flow into the cavity) that in turn may increase the precision of the thermometry. Such improvements will be evaluated on the ex vivo heart model prior to in vivo validation on animal. 


\section{REFERENCES}

1- World Health Organization. Fact Sheet no 310: Global Burden of Disease Update. Geneva, Switzerland: World Health Organization; 2008.

2- Cleland JGF, Daubert JC, Erdmann E et al. Longer-term effects of cardiac resynchronisation therapy on mortality in heart failure [The Cardiac Resynchronisation-Heart Failure (CARE-HF) trial extension phase]. Eur Heart J 2006;27:1928-1932

3- Calvert M, Freemantle N, Yao G et al. Cost-effectiveness of cardiac resynchronisation therapy: results from the CARE-HF trial. Eur Heart J 2005;26:2681-2688

4- Aidelsburger, P., Grabein, K., Klauss, V. \& Wasem, J. Cost-effectiveness of cardiac resynchronization therapy in combination with an implantable cardioverter defibrillator (CRT-D) for the treatment of chronic heart failure from a German health care system perspective. Clin Res Cardiol 97, 89-97 (2008). Hynynen K. Ultrasound for drug and gene delivery to the brain. Adv Drug Deliv Rev. 2008;60:1209-1217.

5- Hynynen K. MRI-guided focused ultrasound treatments. Ultrasonics. 2009;50:221229

6- Vergara GR, Vijayakumar S, Kholmovski EG, Blauer JJ, Guttman MA, Gloschat C, et al. Real-time magnetic resonance imaging-guided radiofrequency atrial ablation and visualization of lesion formation at 3 Tesla. Heart Rhythm. 2011;8:295-303

7- Black MJ, and Anandan P. A framework for the robust estimation of optical flow. ICCV 1993;231-236

8- Emilien A, Benois-Pineau J, Elbes D, Quesson B, Adaptive rejection of outliers for robust motion compensation in cardiac MR-thermometry, ICIP 2013, Melbourne, Australia.

9- Vigen KK, Daniel BL, Pauly JM, Butts K. Triggered, navigated, multibaseline method for proton resonance frequency temperature mapping with respiratory motion. Magn Reson Med 2003;50:1003-1010.

10- Denis de Senneville B., Roujol S., Jaïs P., Moonen C. T. W., Herigault G., Quesson B., Feasibility of fast MR-thermometry during cardiac RF ablation, Nuclear Magnetic Resonance in Biomedicine, 2012;25(4):556-562.

11-de Senneville BD, Ries M, Maclair G, Moonen C. MR-guided thermotherapy of abdominal organs using a robust PCA-based motion descriptor. IEEE Trans Med Imaging 2011:30;1987-1995.

12- C. Sanderson, Armadillo: An Open Source C++ Linear Algebra Library for Fast Prototyping and Computationally Intensive Experiments, Technical Report, NICTA, 2010

13- Vaillant F, Magat J, Naulin J, Loyer V, Vieillot D, Deschodt-Arsac V, et al. Development of a MR-compatible ex vivo working pig heart setup for structural and functional analysis of cardiac diseases. ISMRM, Milan, Italy 2014. 



\section{FIGURE CAPTIONS}

Figure 1. Cardiac MR-thermometry real-time acquisition-processing pipeline

a) Framework for real-time MR-thermometry.

b) Acquisition scheme using ECG triggering and free breathing.

c) \& d) Comparison of the temperature standard deviation (TSD) on healthy volunteers: Box plots ( $25 \%$, median, $75 \%$ ) of the TSD distribution in the ROIs located in the heart, TSD images overlaid on magnitude images (volunteer \#5) for the two correction methods.

e) MR Thermometry results obtained on an isolated working pig heart: The temporal evolution of the temperature near the distal electrode (left). Catheter (in red) and approximate slice position (in green) (right).

\section{Figure 2. Ex vivo working heart results}

Cardiac response to electrical pacing applied on the right atrium. LV pressures (top trace), and electrical signals for RA (middle trace), LV (bottom trace) are reported during atrial pacing. Short axis CINE images (bottom image extracted from the time series) could be collected during pacing without image degradation.

\section{Figure 3. EP recordings in absence (a) or during (b) MRI scanning.}

Traces in (a) display EP signals simultaneously recorded by the 2 catheters (voltage measured between the distal (D) and the second (2) electrodes) located in the RA (top) and in the LV (bottom). During MRI-thermometry scanning (b), the signals recorded in the RA between D-2 and D-4 display artifacts (arrows) although hardware filters (top traces) were inserted into the transmission lines. Additional digital low-pass filtering (bottom traces) of each signal shows substantial reduction of artifacts amplitude. 
a) Framework for real-time MR-thermometry

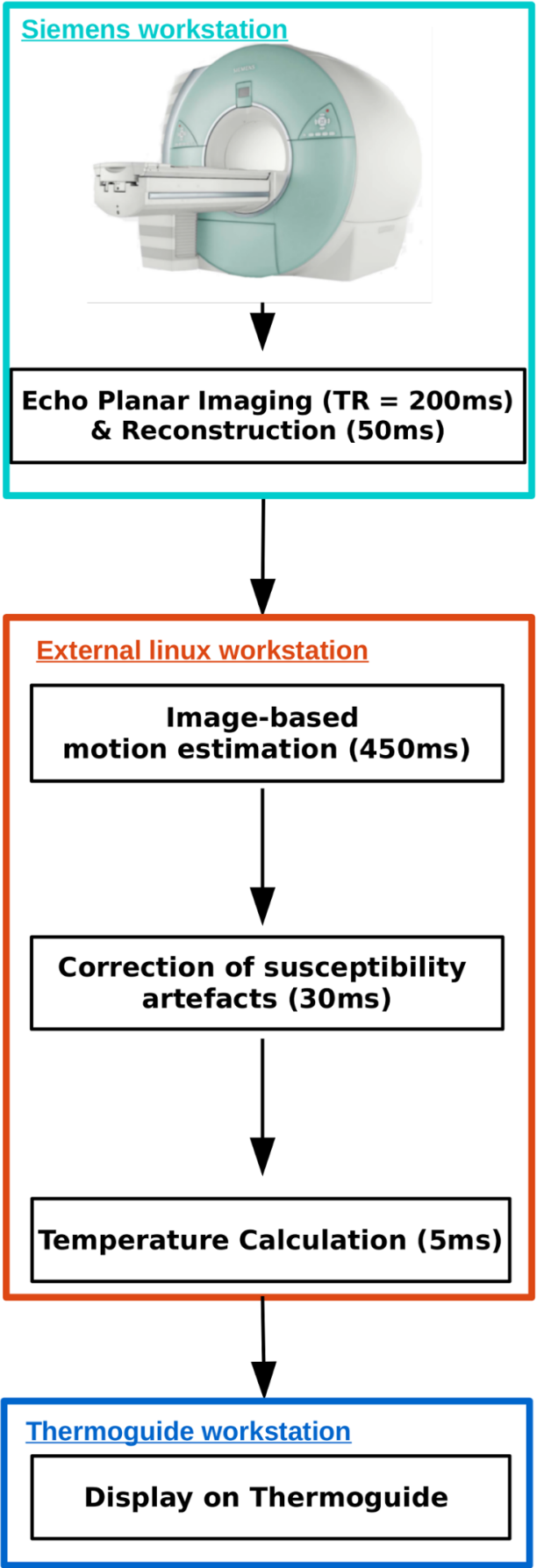

b) Acquisition scheme using ecg triggerring

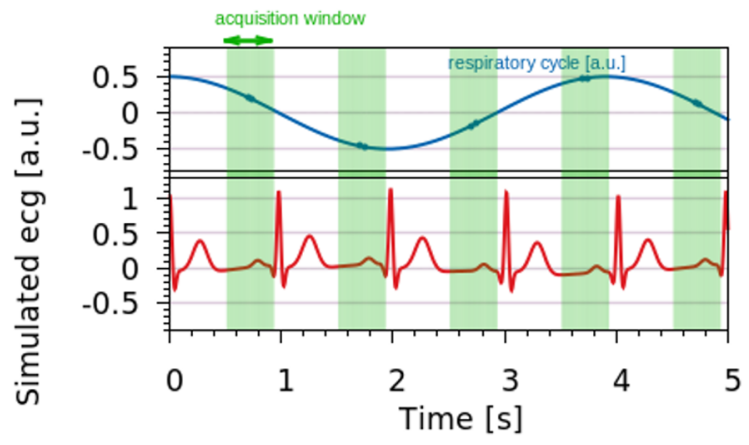

c) Statistical analysis of temperature

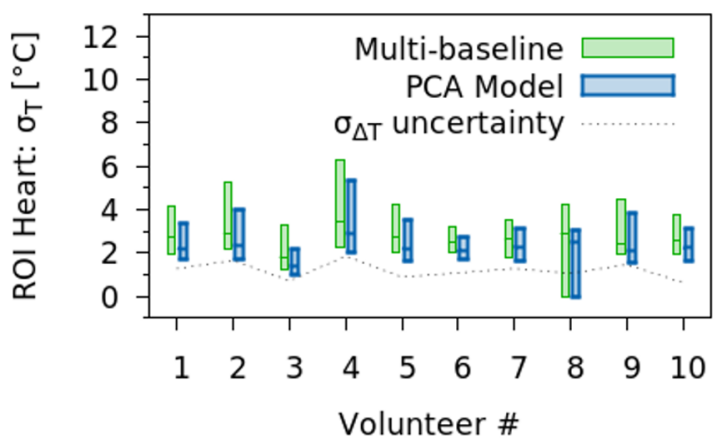

d) Temporal standard deviation of temperature (TSD)

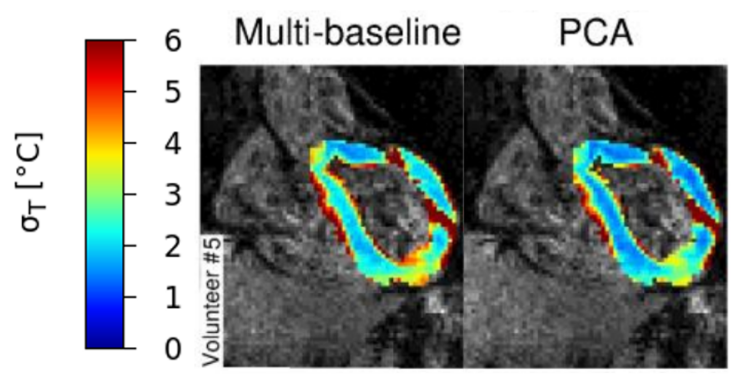

e) Temperature rise during catheter ablation

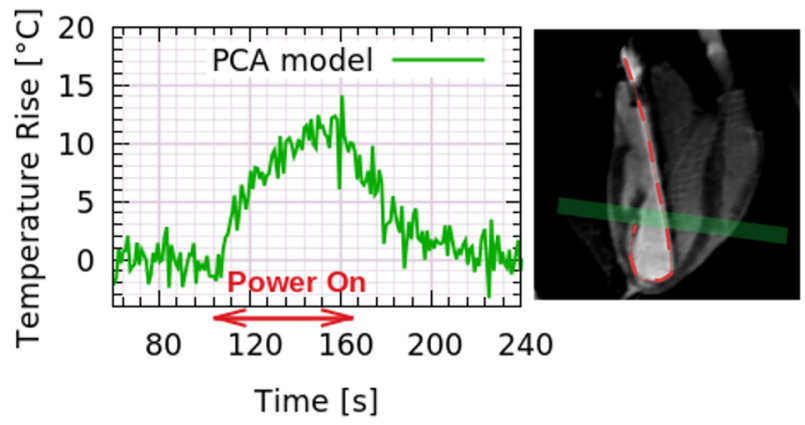


Figure_2 LV Pressure

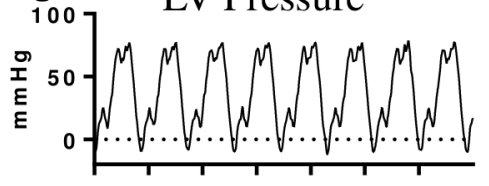

RA electrical signal

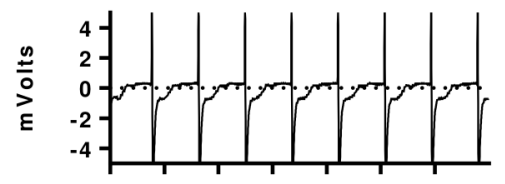

LV electrical signal

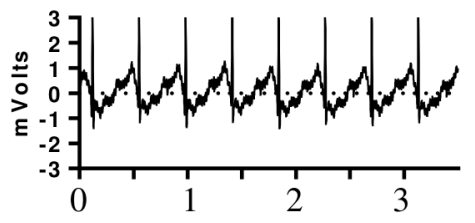

Time / sec

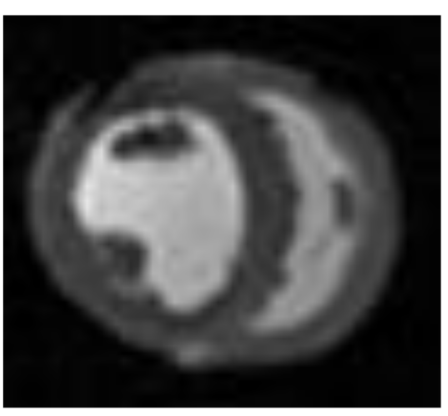


Figure_3
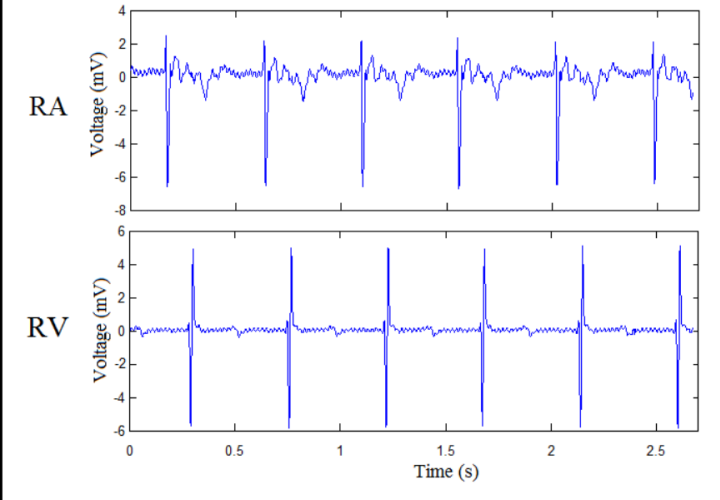

b

D-2

Unfiltered

Filtered

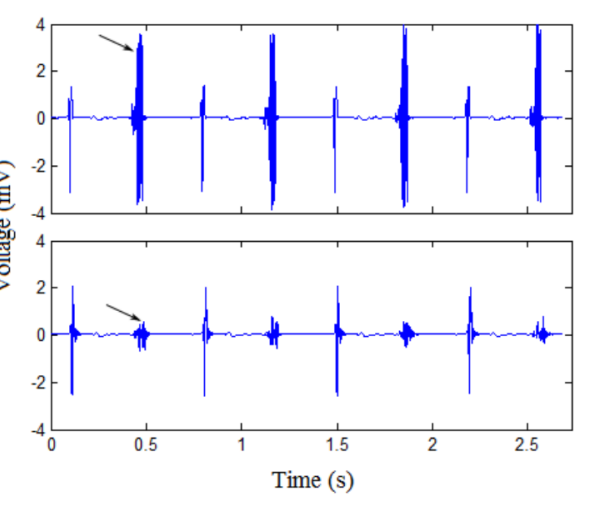

\section{D-4}

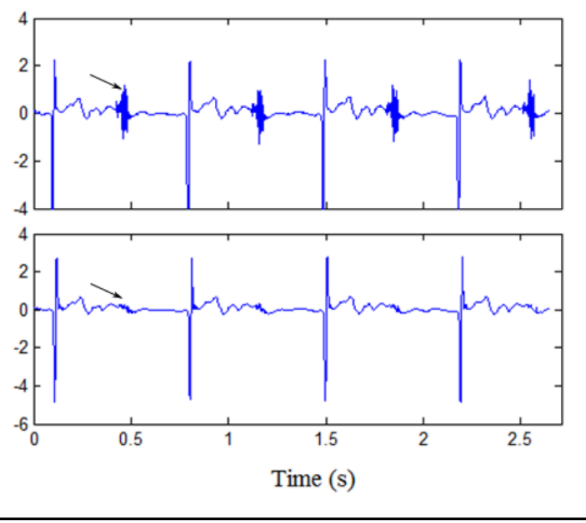

